\title{
Investigating effects of different artefact types on motor imagery $\mathrm{BCl}$
}

\author{
Frølich, Laura; Winkler, Irene; Muller, Klaus-Robert; Samek, Wojciech
}

Published in:

Proceedings of the 2015 37th Annual International Conference of the IEEE Engineering in Medicine and Biology Society (EMBC)

Link to article, DOI:

10.1109/EMBC.2015.7318764

Publication date:

2015

Document Version

Peer reviewed version

Link back to DTU Orbit

Citation (APA):

Frølich, L., Winkler, I., Muller, K-R., \& Samek, W. (2015). Investigating effects of different artefact types on motor imagery BCl. In Proceedings of the 2015 37th Annual International Conference of the IEEE Engineering in Medicine and Biology Society (EMBC) (pp. 1942-1945). IEEE. I E E E Engineering in Medicine and Biology Society. Conference Proceedings https://doi.org/10.1109/EMBC.2015.7318764

\section{General rights}

Copyright and moral rights for the publications made accessible in the public portal are retained by the authors and/or other copyright owners and it is a condition of accessing publications that users recognise and abide by the legal requirements associated with these rights.

- Users may download and print one copy of any publication from the public portal for the purpose of private study or research.

- You may not further distribute the material or use it for any profit-making activity or commercial gain

- You may freely distribute the URL identifying the publication in the public portal 
(C)2015 IEEE. Personal use of this material is permitted. Permission from IEEE must be obtained for all other uses, in any current or future media, including reprinting/republishing this material for advertising or promotional purposes,creating new collective works, for resale or redistribution to servers or lists, or reuse of any copyrighted component of this work in other works. http://ieeexplore.ieee.org/xpl/ articleDetails.jsp?arnumber $=7318764$ 


\title{
Investigating effects of different artefact types on Motor Imagery BCI
}

\author{
Laura Frølich ${ }^{1}$, Irene Winkler ${ }^{2}$, Klaus-Robert Müller ${ }^{3}$, Member, IEEE, and Wojciech Samek ${ }^{4}$, Member, IEEE
}

\begin{abstract}
Artefacts in recordings of the electroencephalogram (EEG) are a common problem in Brain-Computer Interfaces (BCIs). Artefacts make it difficult to calibrate from training sessions, resulting in low test performance, or lead to artificially high performance when unintentionally used for BCI control. We investigate different artefacts' effects on motorimagery based BCI relying on Common Spatial Patterns (CSP). Data stem from an 80-subject BCI study. We use the recently developed classifier IC_MARC to classify independent components of EEG data into neural and five classes of artefacts. We find that muscle, but not ocular, artefacts adversely affect BCI performance when all 119 EEG channels are used. Artefacts have little influence when using 48 centrally located EEG channels in a configuration previously found to be optimal.
\end{abstract}

\section{INTRODUCTION}

Brain-Computer Interfaces (BCIs) allow a user to control a computer through his or her brain activity. The brain activity is often examined using electroencephalography (EEG) recordings, which offer a high temporal resolution and can be acquired with relatively low-cost, transportable equipment.

EEG signals show fluctuations of electrical acitivity as measured from electrodes placed on the scalp. These are also affected by electrical sources unrelated to brain activity, referred to as artefacts, which often produce larger potential differences than brain activity. Some artefacts are of physiological origin, such as eye movements, muscle contractions, the heartbeat etc. while others, such as loose electrodes and the power grid, are technical artefacts.

\section{A. Motivation}

An often cited goal of BCIs is to enable paralysed patients to communicate. Since healthy subjects are easier to recruit, development of BCIs is usually carried out on healthy subjects. If a BCI system developed on healthy subjects turns out to be controlled by artefacts, it will be of little use

\footnotetext{
*This work was supported by the Federal Ministry of Education and Research (BMBF) under the project Adaptive BCI (FKZ 01GQ1115) and by the Brain Korea 21 Plus Program through the National Research Foundation of Korea funded by the Ministry of Education.

${ }^{1}$ Laura Frølich is with the Section for Cognitive Systems, DTU Compute, Technical University of Denmark, Matematiktorvet, Building 321, $2800 \mathrm{Kgs}$. Lyngby, Denmark lffredtu.dk

Irene Winkler is with the Machine Learning Group, Technische Universität Berlin, Marchstr. 23, 10587 Berlin, Germany irene.winkleratu-berlin. de

${ }^{3}$ Klaus-Robert Müller is with the Machine Learning Group, Technische Universität Berlin, Marchstr. 23, 10587 Berlin, Germany and also with the Department of Brain and Cognitive Engineering, Korea University, Seoul 136-713, Republic of Korea klaus-robert.muelleratu-berlin.de

${ }^{4}$ Wojciech Samek is with the Machine Learning Group, Fraunhofer Heinrich Hertz Institute, Einsteinufer 37, 10587 Berlin, Germany wojciech.samek@hhi.fraunhofer.de
}

in patients. Even if a BCI system is developed for healthy subjects, artefacts may be problematic if the stimulus during training induces other artefacts than those from online use.

Some artefacts may affect BCI training more than others and the methods for remedying different artefacts' effects differ. By investigating artefacts' influence on BCIs we aim to identify those most detrimental to performance which can then be targeted to gain the largest improvements.

\section{B. Previous work on artefacts' effects on BCIs}

Only few studies have previously inspected the influence of artefacts on motor-imagery based BCIs. McFarland et al. inspected the presence of muscle artefacts in $10 \mathrm{BCI}$ sessions of novices [1]. Muscle artefacts either caused or indicated frustration with lacking BCI control. Winkler et al. investigated the performance of a motor-imagery based BCI system as a function of the number of removed artefactual data dimensions [2]. No substantial decrease in performance was observed until fewer than 12 dimensions remained in training data. Others have proposed variations of Common Spatial Patterns (CSP) to cope with artefacts [3], [4], [5], [6], [7]. To the best of our knowledge, no study has previously attempted to quantify the influence of different types of artefacts on motor-imagery based BCI.

\section{Aim and research questions}

We wish to learn how various artefact types affect motorimagery based BCI systems. Using data from an 80-subject BCI study, we applied Independent Component Analysis (ICA) to linearly transform EEG signals into a space of independent source components (ICs). We then used the recently developed multi-class classifier 'IC_MARC' to label each component as neural activity or as one of five artefact types (blinks, lateral eye movements, heartbeat artefact, muscle artefact, or mixed artefact) [8]. Mixed artefacts are artefacts that do not clearly belong to one of the other four artefact classes and may also include traces of neural activity. We answered the following research questions:

1) What types of artefacts are most common in training data (after automatic removal of noisy channels)?

2) Do participants use information contained in artefactual ICs to control the BCI system?

3) Does removing or regularising away from artefactual ICA directions improve BCI performance?

4) Do the answers for the above questions differ depending on whether all available EEG channels (119 channels) or only the 48 central channels found to be optimal by Sannelli et al. [9] are used? 


\section{Methods \& MAterials}

\section{A. Data}

Data stem from Blankertz et al. [10], who recorded 80 BCI-novices in a classical motor-imagery paradigm. Subjects were paid 8 EUR per hour for participation [10]. Participants first performed motor imagery with the left hand, right hand and both feet in a training measurement. Every $8 \mathrm{~s}$, the requested BCI task of the current trial was indicated by a visual cue. Following calibration of the system, the test data were recorded using the two classes that provided best discrimination. Participants controlled a 1D cursor application. For the training data 75 trials for each motor condition were recorded, while the test data contained 150 trials from each condition. All BCI performance tests were performed on test data for each participant, while ICA demixing and training of the BCI-classifier were based on calibration data.

EEG data were recorded from 119 electrodes placed according to the extended 10-20 system at a frequency of $1000 \mathrm{~Hz}$. For our offline re-analysis, data were band-pass filtered between $8-30 \mathrm{~Hz}$. Epochs were defined as $0.75-3.5 \mathrm{~s}$ after event markers. In the training data, channels with excessively low or high variance were automatically rejected.

\section{B. Determining effects of artefacts on BCI performance}

1) Common Spatial Patterns: Common Spatial Patterns is a standard feature extraction method for motor-imagery based BCIs [11]. CSP extracts spatial filters as linear channel combinations, w, for which the variance differs most between conditions. Formally, CSP filters are the eigenvectors corresponding to the largest (and smallest) eigenvalues $\lambda$ of the generalized eigenvalue problem $C_{1} \mathbf{w}=\lambda C_{2} \mathbf{w}$, found as:

$$
\underset{\mathbf{w}}{\operatorname{argmax}} \frac{\mathbf{w}^{T} C_{1} \mathbf{w}}{\mathbf{w}^{T} C_{2} \mathbf{w}} .
$$

The channel $\times$ channel matrix $C_{i}$ is the average of covariance matrices from condition $i$ trials. We used the filters from the three highest and lowest eigenvalues for classification.

2) Automatic classification of independent components: For each subject, we ran an ICA on the concatenated training data epochs. We used the extended Infomax algorithm in EEGLab [12] to extract enough ICs to account for $99.9 \%$ of data variance. Each IC consists of its time course and a spatial pattern which expresses the IC's influence on scalp electrodes. Subsequently, we used the previously developed automatic classifier "IC_MARC" to classify ICs [8].

IC_MARC uses multinomial regression to assign probabilities to ICs of belonging to each of six classes (blinks, lateral eye movements, electrical heartbeat, muscle, neural, or mixed artefact). We used features of the scalp maps for classification. This is, to the best of our knowledge, the only existing classifier allowing distinction between both ocular and muscular artefacts. Most other classifiers can distinguish between different ocular, but not muscular artifacts (e.g. [13], [14]), or cannot be used in a multi-class setting.

ICs were classified as belonging to the class for which the highest probability was predicted, except if the highest probability was for an ocular artefact class and that probability was less than $80 \%$. Such ICs were classified as mixed. Fig. 1 shows patterns from ICs classified by IC_MARC. ${ }^{1}$

For the analysis presented here, we consider three groups of artefactual ICs: 1) muscle artefacts, 2) ocular artefacts (eye blink and horizontal eye movements), and 3) all nonneural components (eye blink, electrical heartbeat, lateral eye movement, muscle, and mixed artefacts).

3) EEG channel configuration: If only central channels are kept it is likely that some artefacts become less pronounced or disappear, as e.g. muscle artefacts affect outer electrodes most (see Fig. 1). Since artefacts may affect electrode configurations differently we analysed both the full electrode configuration and the electrode configuration found to be optimal by Sannelli et al. that consists of 48 centrally located electrodes [9].

4) BCI performance on artefactual and non-artefactual data: We applied CSP to the activity contained in artefactual ICs to quantify the amount of class-discriminative information in artefacts. We also investigated the BCI performance when different groups of artefacts were projected out.

5) BCI performance when artefacts are regularised against: Since artefactual ICs may contain traces of neural activity, we might expect CSP performance to increase when we regularise against artefactual directions instead of completely removing them. This should allow the CSP algorithm to find spatial filters in the artefactual directions if there is enough class-discriminative information to warrant this.

By introducing a channel $\times$ channel regularisation matrix $K$ (and a regularisation parameter $\lambda \in \mathbb{R}$ ) in the CSP objective as follows, spatial filters that cause large variance along the directions of $K$ are discouraged [15]:

$$
\underset{\mathbf{w}}{\operatorname{argmax}} \frac{\mathbf{w}^{T} C_{1} \mathbf{w}}{\mathbf{w}^{T}\left((1-\lambda) C_{2}+\lambda K\right) \mathbf{w}} .
$$

To regularise against artefactual directions, normalised patterns of artefactual ICs were collected as columns in a matrix, $A_{\text {art }}$. Analyses not reported here showed no significant difference in performance between making patterns or time series of ICs have norm one.

The penalty matrix $K$ was set equal to $A_{\text {art }} A_{\text {art }}^{T}$ to find spatial filters w such that $\left\|\mathbf{w}^{T} A_{\text {art }}\right\|$ is minimal, where $\|\cdot\|$ denotes the euclidean norm. This choice can be understood by looking at the ICA decomposition of the EEG data $X$, given as $X=A_{\text {art }} S_{\text {art }}+A_{\text {neuro }} S_{\text {neuro }}$, where $S$ contains the time courses of ICs in rows and the subscript neuro denotes neural ICs. The source activity extracted by a spatial filter $\mathbf{w}$, given as $\mathbf{w}^{\top} X$, contains minimal contributions from artefactual activity $S_{a r t}$ if $\left\|\mathbf{w}^{T} A_{\text {art }}\right\|$ is minimized. (For more information on the interpretation of patterns and filters we refer the reader to [16].)

For each subject, the regularisation parameter $\lambda$ was chosen in a five-fold cross-validation on calibration data from the values $0,2^{-16}, 2^{-15}, \ldots, 2^{-1}, 0.6,0.7, \ldots, 1$.

\footnotetext{
${ }^{1}$ Except for the heartbeat class, the examples are good demonstrations of what one would expect in each class. Difficulty with the heartbeat class was also found during the development of IC_MARC and CORRMAP [8], [13].
} 


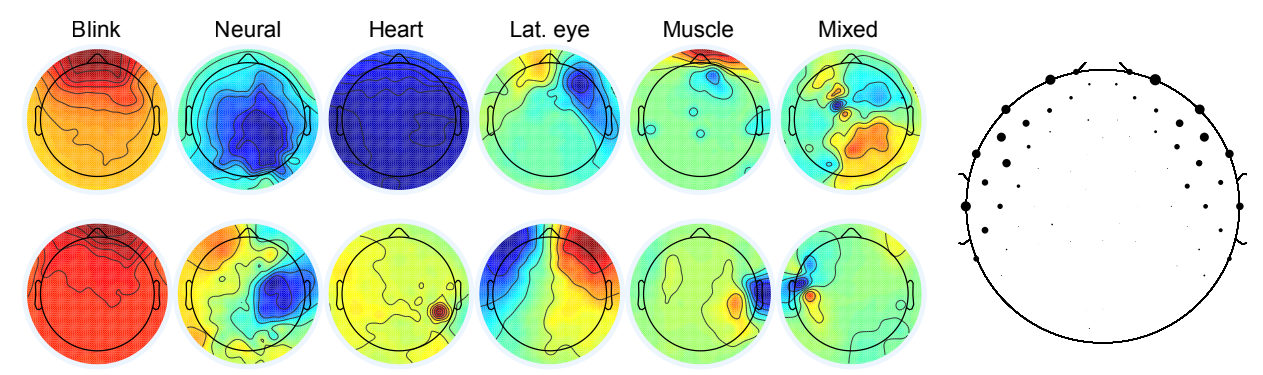

Fig. 1: Left: Examples of patterns of automatically classified ICs. Right: Locations of most active electrode in muscle ICs from all subjects. Dot sizes represent the number of times electrodes were the $\overline{\text { most }}$ active in muscle ICs.

\section{RESULTS}

\section{A. Most common artefacts}

Mixed and muscle artefacts were the most and second most common artefact classes, respectively. Using all channels, out of 6428 (range over subjects: 39-107) ICs, 33 (0-14) were classifed as blinks, 1854 (4-43) as neural, $57(0-4)$ as heartbeats, $80(0-9)$ as lateral eye movements, 1773 (8-45) as muscular, and 2631 (5-86) as mixed. On the 48 channels, out of 2925 (range: 22-45) ICs, 7 (0-4) were classifed as blinks, 1320 (6-25) as neural, $21(0-4)$ as lateral eye movements, $276(0-12)$ as muscular, and 1301 (5-33) as mixed.

\section{B. Class-discriminative information in artefacts}

Using the Wilcoxon signed rank test we found that error rates significantly differed from chance $(50 \%)$ when CSP was trained on muscular or all non-neural ICs $(p<0.0001$, both channel configurations). When trained on ocular artefacts, the performance did not differ from chance ( $p$-values of 0.39 and 0.75 for the all- and 48-channel configurations, respectively). This shows that only the muscle and non-neural artefact groups contain class-discriminative information.

We used a sign test to compare the performance for each subject when muscle artefacts were removed to the baseline by looking at whether each trial was correctly or incorrectly classified. On the full channel configuration, the performance of 19 subjects significantly changed when muscle artefacts were removed, 6 getting worse. On the 48-channel configuration the performance of 17 subjects changed, 9 getting worse. When removing all non-neural ICs, the performance of 12 and 17 subjects significantly decreased on the all-channel and 48-channel configurations while 10 and 12 subjects improved on the two configurations, respectively.

\section{Does removing or regularising away from artefactual ICA directions improve BCI performance?}

Table I shows error rates obtained from baseline CSP, CSP trained on non-artefactual activity, and CSP with artefact regularisation for all three artefact groups. Significance tests were calculated using the Wilcoxon signed rank test. Since subjects with the same performance in two methods are not included in the comparision, some differences between medians may be higher than others without showing corresponding significance. On the full channel configuration, the only significant difference from baseline CSP was obtained when regularising against muscle ICs, which improved performance. Removing muscle ICs did not result in a significant difference from the CSP baseline although the median performance was better than that obtained with regularisation. This shows that regularising gives a more consistent improvement across subjects. With regularisation, however, artefactual activity could still be used to gain artificially high levels of BCI control.

On the 48-channel configuration muscle artefacts were not as prominent, which is reflected by the lack of performance improvement with regularisation against muscle ICs. In line with the observation that ocular artefacts did not contain class-discriminative information for either channel configuration, we observed that regularising against or removing ocular ICs did not significantly impact performance.

Fig. 2 shows the relationship between improvements in performance when removing non-neural ICs and the CSP classification performance when training only on those non-neural ICs, on the 48-channel configuration. A higher error rate from training on artefacts implies less classdiscriminative information in the artefacts. Hence removing such artefacts should make the neural signal clearer without removing class-relevant data. This is indeed what the figure shows since the improvement with artefact removal increases with the error rate from training on artefacts.

When artefacts contain class-discriminative information it could be due to traces of neural activity in the artefacts or to the user employing artefacts to control the BCI. Fig. 3 shows

TABLE I: Error Rates

\begin{tabular}{|c|c|c|c|}
\hline & Muscle & Ocular & All non-neural \\
\hline \multicolumn{4}{|l|}{48 channels } \\
\hline CSP & - & $-28.25 \quad(1.7)$ & \\
\hline CSP no artefacts & 27.17 & 27.67 (1.7) & $28.50 *(1.7)$ \\
\hline CSP IC regularised & $29.50 *(1.7)$ & $29.17 \quad(1.7)$ & $28.83 *(1.7)$ \\
\hline \multicolumn{4}{|l|}{ All channels } \\
\hline CSP & - & -31.75 (1.8) & \\
\hline CSP no artefacts & $29.08 \quad(1.8)$ & 32.33 (1.8) & $35.00 \quad(1.7)$ \\
\hline CSP IC regularised & $31.42 *(1.8)$ & $33.50 \quad(1.8)$ & $32.00 \quad(1.8)$ \\
\hline
\end{tabular}

Median error rates over 80 subjects from baseline CSP, CSP trained on non-artefactual activity, and CSP with artefact regularisation for three artefact groups (standard deviations in parentheses). * indicates differences from baseline CSP $(p<0.05)$. 
an example indicating that artefacts were used to control the BCI since performance decreased after artefact removal and muscular artefact contamination is seen in the last two CSP patterns before artefact removal but not after.

\section{CONCLUSION}

We investigated the influence of different artefacts on motor-imagery based BCIs. Using data from an 80-subject BCI study and a recently proposed multi-class IC classifier, we found that muscle artefacts alone and all non-neural artefacts as a group have a small impact on the BCI system. In contrast, ocular artefacts alone had no significant influence, probably because eye artefacts mostly affect frequency ranges below those containing the motor-imagery $\mu$-rhythm.

More specifically, we observed above-chance performance when CSP was trained on muscular or mixed artefacts, but not if trained on ocular artefacts. Up to 9 subjects used muscle artefacts to improve their BCI control. This may be problematic if healthy participants use artefacts to operate a BCI system which should be transferable to severely motor-impaired patients. However, we note that the overall contribution of muscle artefacts was significant, but small.

When removing artefacts, BCI performance did not improve, which is consistent with previously published analyses that used a different automatic classifier of ICs [2], [17]. Regularising against muscle artefacts significantly improved BCI performance when all available 119 channels were used but significantly impaired performance for the more suitable 48-channel configuration.

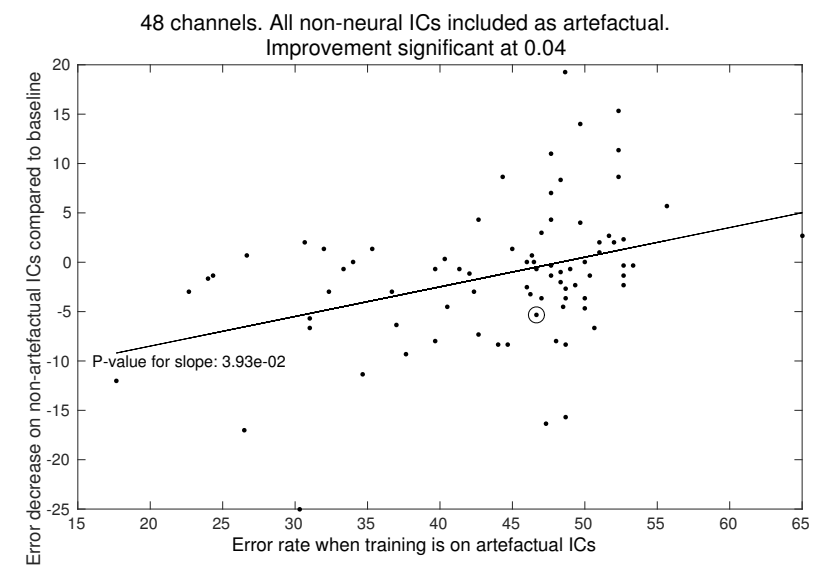

Fig. 2: Error decrease when CSP was run on neural ICs relative to the performance on artefactual ICs. The circled dot represents the subject whose CSP patterns are shown in Fig. 3.

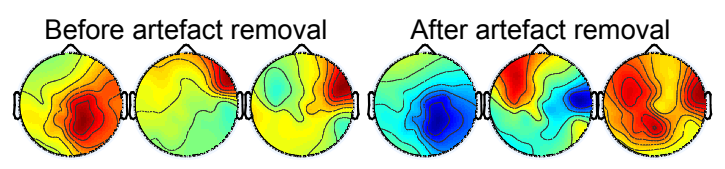

Fig. 3: The three most discriminative CSP patterns for class one from all data and neural ICs only on the all-channel configuration. Artefact removal increased the error rate from $11.67 \%$ to $17 \%$.
We conclude that it is difficult to improve CSP performance on the 48-channel configuration by artefact processing. We conjecture that this difficulty may mainly arise from using the motor-imagery paradigm which relies on activity in the motor cortices, recorded from central scalp positions.

\section{Acknowledgment}

We thank the authors of [10] for providing data and Claudia Sannelli for advise on optimal electrode configurations.

\section{REFERENCES}

[1] D. J. McFarland, W. A. Sarnacki, T. M. Vaughan, and J. R. Wolpaw, "Brain-computer interface (bci) operation: signal and noise during early training sessions," Clinical Neurophysiology, vol. 116, no. 1, pp. $56-62,2005$.

[2] I. Winkler, S. Haufe, and M. Tangermann, "Automatic classification of artifactual ICA-components for artifact removal in EEG signals," Behavioral And Brain Functions, vol. 7, p. 30, 2011.

[3] W. Samek, M. Kawanabe, and K.-R. Müller, "Divergence-based framework for common spatial patterns algorithms," IEEE Reviews in Biomedical Engineering, vol. 7, pp. 50-72, 2014.

[4] M. Kawanabe, W. Samek, K.-R. Müller, and C. Vidaurre, "Robust common spatial filters with a maxmin approach," Neural Computation, vol. 26, no. 2, pp. 1-28, 2014.

[5] F. Lotte and C. Guan, "Regularizing common spatial patterns to improve BCI designs: unified theory and new algorithms," IEEE Transactions On Biomedical Engineering, vol. 58, no. 2, pp. 355-362, Feb 2011.

[6] B. Reuderink, "Robust brain-computer interfaces," Ph.D. dissertation, University of Twente, Enschede, The Netherlands, October 2011.

[7] W. Samek, C. Vidaurre, K.-R. Müller, and M. Kawanabe, "Stationary common spatial patterns for brain-computer interfacing," Journal of Neural Engineering, vol. 9, no. 2, p. 026013, 2012.

[8] L. Frølich, T. S. Andersen, and M. Mørup, "Classification of independent components of eeg into multiple artifact classes," Psychophysiology, vol. 52, no. 1, pp. 32-45, Jan 2015.

[9] C. Sannelli, T. Dickhaus, S. Halder, E.-M. Hammer, K.-R. Müller, and B. Blankertz, "On optimal channel configurations for smr-based braincomputer interfaces," Brain Topography, vol. 23, no. 2, pp. 186193, 2010.

[10] B. Blankertz, C. Sannelli, S. Halder, E. M. Hammer, A. Kübler, K. R. Müller, G. Curio, and T. Dickhaus, "Neurophysiological predictor of SMR-based BCI performance," Neuroimage, vol. 51, no. 4, pp. 13031309, Jul 2010.

[11] B. Blankertz, R. Tomioka, S. Lemm, M. Kawanabe, and K.-R. Müller, "Optimizing spatial filters for robust EEG single-trial analysis," IEEE Signal Processing Magazine, vol. 25, no. 1, pp. 41-56, Jan 2008.

[12] A. Delorme and S. Makeig, "Eeglab: an open source toolbox for analysis of single-trial EEG dynamics including independent component analysis," Journal of Neuroscience Methods, vol. 134, pp. 9-21, 2004.

[13] F. C. Viola, J. Thorne, B. Edmonds, T. Schneider, T. Eichele, and S. Debener, "Semi-automatic identification of independent components representing EEG artifact." Clinical Neurophysiology, vol. 120, no. 5, pp. 868-77, 2009.

[14] A. Mognon, J. Jovicich, L. Bruzzone, and M. Buiatti, “Adjust: An automatic eeg artifact detector based on the joint use of spatial and temporal features," Psychophysiology, vol. 48, no. 2, pp. 229-240, 2010.

[15] B. Blankertz, M. K. R. Tomioka, F. U. Hohlefeld, V. Nikulin, and K.-R. Müller, "Invariant common spatial patterns: Alleviating nonstationarities in brain-computer interfacing," in Advances in Neural Information Processing 20. MIT Press, 2008, p. 2008.

[16] S. Haufe, F. Meinecke, K. Görgen, S. Dähne, J.-D. Haynes, B. Blankertz, and F. Bießmann, "On the interpretation of weight vectors of linear models in multivariate neuroimaging," NeuroImage, vol. 87, no. 0, pp. $96-110,2014$.

[17] I. Winkler, S. Brandl, F. Horn, E. Waldburger, C. Allefeld, and M. Tangermann, "Robust artifactual independent component classification for bci practitioners," Journal of Neural Engineering, vol. 11, no. 3, p. 035013, 2014. 